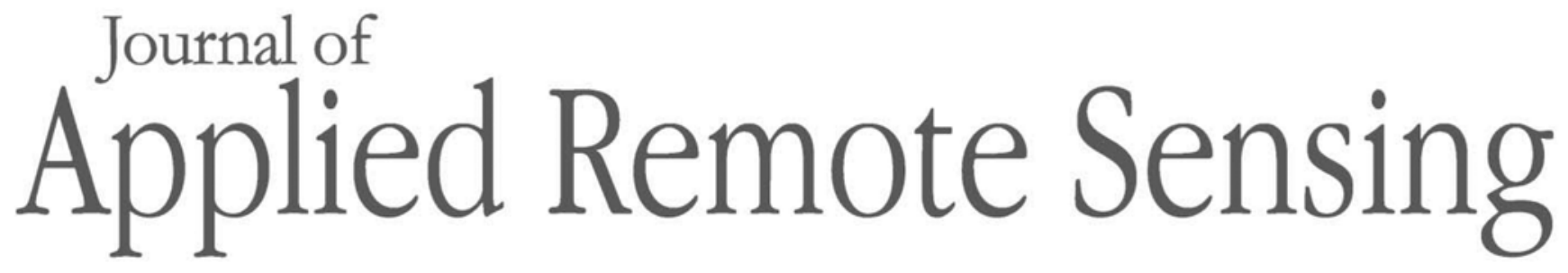

RemoteSensing.SPIEDigitalLibrary.org

\title{
Special Section Guest Editorial: Advances in Deep Learning for Hyperspectral Image Analysis and Classification
}

\author{
Masoumeh Zareappor \\ Jinchang Ren \\ Huiyu Zhou \\ Wankou Yang
}




\title{
Special Section Guest Editorial: Advances in Deep Learning for Hyperspectral Image Analysis and Classification
}

\author{
Masoumeh Zareappor, ${ }^{\text {a Jinchang Ren, }}{ }^{\text {b }}$ Huiyu Zhou, ${ }^{c}$ and \\ Wankou Yang \\ a'Shanghai Jiao Tong University, Institute of Image Processing and Pattern Recognition, \\ Shanghai, China \\ ${ }^{b}$ University of Strathclyde, Strathclyde Hyperspectral Imaging Centre, \\ Department of Electronic and Electrical Engineering, Glasgow, Scotland, United Kingdom \\ 'University of Leicester, Knowledge Discovery and Machine Learning Theme, \\ Biomedical Image Processing Lab, Leicester, England, United Kingdom \\ ${ }^{\mathrm{d}}$ Southeast University, School of Automation, Nanjing, China
}

Remote sensing is a classical area of research that has been involved in many crucial applications, including urban development agriculture, scene interpretation, defense, weather, and other non-Earth observations. In the last decade, the analysis of hyperspectral images (HSIs) acquired by remote sensors has gained substantial attention and is increasingly becoming an active research discipline. However, there are some main challenges in hyperspectral data classification, such as ultra-high dimensionality of data, a limited number of labeled instances, and large spatial variability of spectral signature. These challenges degrade the ability to differentiate the pairwise distance between points and make it difficult to discriminate the most relevant features, causing the classification performance to give wrong or inaccurate results. Therefore, in processing hyperspectral images, the classification approaches have been proposed jointly by dimensionality reduction. Several feature extraction based HSI have been developed to solve the classification problem in hyperspectral images. These methods aim to reduce the dimensionality of the data while preserving the discriminative information of both spectral and spatial features.

Deep learning has become the de facto for many computer vision tasks. However, hyperspectral image classification and analysis is still in its infancy, so there are few works on the concept. In part, this is because remote sensing poses unique challenges, such as lack of labeled training data and diversity features (spectral-spatial). Whereas we are excited about the potential of deep learning for remote sensing, we are equally nervous about whether this technology can deliver. Furthermore, it is an analytics tool to help us better understand these sensors, platforms, and applications.

In this special section, we requested new application papers on hyperspectral imaging to show what has been done and what is being done. This special section includes seven high quality papers, which in particular address hyperspectral image classification and analysis. One paper is based on multispectral image fusion for HSI, in which the spectral and spatial information of HSI are fused into the same feature map. One paper uses a two-mode method to identify surface water in particular images. Two papers utilize spectral-spatial processing for hyperspectral image analysis. One paper uses multiscale segmentation-based CNN to increase the resolution of the HSI. Two papers are based on sparse representation to achieve temporal-angular fusion.

Since this special section is based on new deep learning methods in HSI, we focused on those papers that rely on new techniques and real datasets. We believe that all of these papers will attract both expert and nonexpert readers. We appreciate the effort of a large number of reviewers, whose work and dedication are here deeply acknowledged. We have special thanks to the following contributors for their great support.

- Prof. Jie Yang, Institute of Image Processing and Pattern Recognition, Shanghai Jiao Tong University, China.

- Prof. Ruili Wang, Massey University, New Zealand.

- Pourya Shamsolmoali, Department of Automation, Shanghai Jiao Tong University, China.

- Fabrizio Antonio, ASC - Advanced Scientific Computing Division, CMCC, Italy. 
Masoumeh Zareappor is an associate researcher at the Institute of Image Processing and Pattern Recognition, Shanghai Jiao Tong University, Shanghai. She recently was recognized as a Young Talented Scientific Researcher by the government of China. She has published more than 30 peer-reviewed articles in international journals. Her research interests include computer vision, image/video analysis and understanding, machine learning, hyperspectral imagery, and visual surveillance.

Jinchang Ren is an associate professor and deputy director of Strathclyde Hyperspectral Imaging Centre, Department of Electronic and Electrical Engineering, University of Strathclyde, UK. His research interests include visual computing, computer vision, contentbased image/video analysis and understanding, machine learning, human-computer interaction, visual surveillance, archive restoration, motion estimation, large scale data analysis and visualization (e.g. hyperspectral imagery and medical data), and intelligent multimedia information processing. His work has been supported by NERC, EPSRC, RSE, NSFC (China), industrial partners, and internal funding.

Huiyu Zhou is an associate professor and heads the Knowledge Discovery and Machine Learning Theme and leads the Biomedical Image Processing Lab at University of Leicester. He has several other duties, including director of MSc programs, coordinator of MSc distance learning projects and member of Research Committee in the Department of Informatics. His research mainly focuses on machine learning, computer vision, and artificial intelligence. $\mathrm{He}$ currently serves as the editor-in-chief of Recent Advances in Electrical \& Electronic Engineering, associate editor of IEEE Transactions on Human-Machine Systems, and editorial board member and guest editor of several refereed journals.

Wankou Yang is an associate professor in the School of Automation, Southeast University, China. His research interests include pattern recognition, machine learning, image processing, and computer vision. He has published more than 80 papers in international peer-reviewed journals, workshops, and conferences. He has served as a guest editor in several journals and also has been organizer and technical chair of several conferences. 